\title{
IDEALS IN HALFRINGS ${ }^{1}$
}

\author{
H. E. STONE
}

\begin{abstract}
An analogue for halfrings of the Hilbert Basis Theorem is obtained by a study of the relationship between the ideals of a halfring and the ideals of its ring of differences. This technique also results in a characterization of those ideals for which the factor semiring has no nontrivial semi-isomorphic images.
\end{abstract}

1. Introduction. Ever since Bourne [3] found it necessary to introduce the concept of a semi-isomorphism of a semiring, it has been of interest to determine when semi-isomorphisms must in fact be isomorphisms. Allen [2] addressed himself to this problem by restricting both the class of ideals and the class of homomorphisms under consideration. In the case of semirings which can be embedded into rings, this paper settles the question without restricting the class of homomorphisms. The basic technique is to associate with each ideal a family of ring ideals which determine the semiisomorphisms having the given ideal as kernel. This focus of attention on the influence of the semiring on its extensions also results in an analogue of the Hilbert Basis Theorem.

A halfring is a triple $(H,+, \cdot)$, where $(H,+)$ is a commutative cancellative semigroup with identity (called zero) and $(H, \cdot)$ is a semigroup whose multiplication distributes over the addition from both sides. A halfring $H$ generates a ring of differences $\bar{H}$ unique up to isomorphism. The operations of $H$ are extended to subsets in the familiar way. Then a subsemiring of $H$ is a subset $S$ with $0 \in S$, with $S+S \subseteq S$, and with $S^{2} \subseteq S$. If also $S H \subseteq S$ or $H S \subseteq S$ or both, then $S$ is a left ideal or right ideal or ideal. As noted in [4], it is not the case that any ideal $K$ of $H$ is the kernel of a homomorphism; for this it is additionally necessary that $h+k \in K$ imply $h \in K$ for each $h \in H$ and each $k \in K$. Such ideals have been called

Presented in part to the Society, January 23, 1969 under the title The Hilbert basis theorem for halfrings and March 17, 1969 under the title $A$ homomorphism theorem for halfrings; received by the editors January 29, 1971.

AMS 1970 subject classifications. Prirnary 16A78.

Key words and phrases. Halfring, Noetherian semiring, Hilbert Basis Theorem, semi-isomorphism, fundamental theorem of homomorphisms.

1 This work is taken from the author's doctoral dissertation at Texas Christian University, directed by Professor Ben T. Goldbeck, Jr. The author thanks Dr. Goldbeck for his advice and encouragement. 
$k$-ideals. A subset $K$ of $H$ having this property will be called subtractive in $H$; thus the $k$-ideals are the subtractive ideals. It is the subtractive ideal which generalizes the notion of ideal in ring theory.

2. Ideals in extension halfrings. Now if $S$ is a subring of $\bar{H}$, it is easy to see that $S \cap H$ is a subtractive subsemiring of $H$. Conversely, if $S$ is a subtractive subsemiring of $H$, the subring $S$ of $\bar{H}$ generated by $S$ is such that $S \cap H=S$. Moreover, these relations continue to hold if subring and subtractive subsemiring are replaced by subtractive right ideal, subtractive left ideal, or subtractive ideal. We summarize these facts in our first theorem.

THEOREM 1. The subtractive subsemirings [right ideals, left idcals, ideals] of a halfring $H$ are precise $y$ the intersections with $H$ of the subrings [subtractive right ideals, subtractive left ideals, subtractive ideals] of its ring of differences $\bar{H}$.

This simple result is of great importance, for it tells us that the ideal structure of a semiring is determined by that of its ring of differences. This relationship is not reciprocal, as the following fundamental example demonstrates.

EXAMPLE. Let $R$ be the ring of all funciions of the nonnegative integers $N$ into the rationals, with the pointwise operations. Let $C$ consist of those functions which are everywhere positive, together with the zero function. Then $C$ is a subsemiring of $R$ and $\bar{C}=R$.

Now each nonzero element of $C$ has a multiplicative inverse, so that $C$ is a division halfring and thus has no nontrivial ideals. But $R$ is a complete direct product of a countably infinite family of copies of the rationals. Hence $R$ does not even satisfy a chain condition on subtractive ideals.

A related problem is to determine the influence of the halfiring ideal structure on other kinds of extensions, polynomial extensions in particular. A surprising relationship appears between the two kinds of extension, as the following discussion will show.

A halfring $H$ is called [right, left] unital if $\bar{H}$ is a ring with [right, left] identity. The set $H[N]$ of all sequences $p: N \rightarrow H$ having only finitely many nonzero terms, when endowed with the usual termwise addition and convolution product, is the halfring of polynomials over $H$. For $n \in N$, it will be convenient to denote the coefficient $(n) p$ by $p_{n}$. It is easily verified that $H[N]$ is a slibsemiiring of $\bar{H}[N]$ consisting of those polynomials over $\bar{H}$ all of whose coefficients lie in $H$, and that $H[N]^{-}=\bar{H}[N]$. Then $H[N]$ is an extension of $H$ when the members of $H$ are identified with the constant polynomials. If $H$ is [right, left] unital, so is $H[N]$. 
Allen [1] has verified the following analogue of a standard result in ring theory:

If $S$ is a subsemiring of a halfring $H$ and $x \in H$ is such that $s x=x s$ for each $s \in S$, then the mapping $(x): S[N] \rightarrow H$ given by $p(x)=\sum_{n} p_{n} x^{n}$ for each $p \in S[N]$ is a homomorphism.

A halfring is [right, left] Noetherian if it satisfies the maximal condition on [right, left] subtractive ideals. Since it is easily seen that the inverse image of a subtractive subsemiring is subtractive, a homomorphic image of a [right, left] Noetherian halfring is [right, left] Noetherian. We are now ready to prove the following generalization of the Hilbert Basis Theorem.

THEOREM 2. Let $H$ be a unital halfring. Then $H[N]$ is right Noetherian if and only if $\bar{H}$ is right Noetherian.

Proof. If $\vec{H}$ is right Noetherian, so is $\bar{H}[N]$ by the classical Hilbert theorem in ring theory. Thus $H[N]$ is right Noetherian by Theorem 1 .

Conversely, suppose $H[N]$ is right Noetherian. Since $H$ is unital and $-1 \in \bar{H}$ commutes with each element of $H,(-1)$ is a homomorphism of $H[N]$ into $\bar{H}$ by Allen's result. Since each element of $\bar{H}$ is of the form $a-b$ for $a, b \in H$, and $a-b=[a+b x](-1), \bar{H}$ is the image of $H[N]$. Thus $\bar{H}$ is right Noetherian. Q.E.D.

The example now shows that an analogue of the Hilbert Basis Theorem does not hold for all halfrings. The class for which it does hold will be called strongly Noetherian-that is, $H$ is strongly Noetherian if $\bar{H}$ is Noetherian. Clearly strongly Ncetherian implies Noetherian.

3. The type of an ideal. The preceding results show the value of a deeper knowledge of the way in which halfring ideals relate to the ideals of the ring of differences. As a tool in this investigation we introduce the concept of ideal type.

Definition. Let $H$ be a halfring, $K$ a subtractive ideal of $H$. The ideal type $\tau(K)$ of $K$ is the set of all subtractive ideals $I$ in $\bar{H}$ such that I $H=K$. If $\tau(K)$ consists of a single ideal, $K$ is said to be ideal monotypic in $H$.

We make similar definitions with ideal replaced by right ideal, left ideal, or subsemiring. Note that $\tau(K)$ depends implicitly on $H$, and that $\tau(H)=$ $\{\bar{H}\}$, so that $H$ is always monotypic in itself. Theorem 1 shows that $\tau(K)$ cannot be empty. In what follows we are interested only in ideal type, which will be called simply type.

THEOREM 3. Let $H$ be a halfring, $\mathscr{K}$ a collection of subtractive ideals of $H$, and $\mathscr{I}$ a collection of ideals of $\bar{H}$ such that (i) $I \in \mathscr{I}$ implies $I \in \tau(K)$ for 
some $K \in \mathscr{K}$ and (ii) for each $K \in \mathscr{K}, \tau(K) \cap \mathscr{I} \neq \varnothing$. Then $\cap \mathscr{I} \in \tau(\bigcap \mathscr{K})$. Further, if $\mathscr{I}$ is a chain, so is $\mathscr{K}$, and $\bigcup \mathscr{I} \in \tau(\bigcup \mathscr{K})$.

COROllary A. If $K$ is a subtractive ideal of a halfring $H$, then $r(K)$ is closed under unions of chains and arbitrary intersections, and contains $R$ as a least member.

Analogues of these results also hold for the other kinds of type discussed above.

The type of 0 in the example $C$ consists of all the proper ideals of $\bar{C}$; hence this is an example of an ideal which is not monotypic. It is also easily seen in this example that $\tau(0)$ is not closed under addition, or even more important, that many pairs of ideals in $\tau(0)$ have no upper bound in $\tau(0)$. Thus the type of an ideal is in general not a lattice.

The importance of ideal type lies in its relation to the collection of homornorphic images of the halfring having given kernel. Recall that a homomorphism $\eta$ of a halfring $H$ into another halfring can be uniquely extended to a homomorphism $\bar{\eta}$ of the respective difference rings by the definition $(a-b) \bar{\eta}=a \eta-b \eta$ for each $a, b \in H$.

THEOREM 4. Let $H$ be a halfring, and let $\eta$ be a ha!fring homomorphism of $H$ with kernel $K$. Let $\bar{\eta}$ be the unique extension of $\eta$ to $\bar{H}$, let $\operatorname{Ker} \bar{\eta}=L$, and let $\lambda$ be the natura! homomorphism of $\bar{H}$ onto $\bar{H} \mid L$. Then $L \in \tau(K)$ and $H \lambda$ is isomorpliic to $H \eta$.

Proof. Clearly $K \subseteq L$. If $h \in L \cap H$, then $h \eta=h \bar{\eta}=0$, and $h \in K$. Thus $L \cap H=K$, and $L \in \tau(K)$.

Now let $\phi=\{(x \lambda, x \eta): x \in H\}$. If $x \lambda=y \lambda$, then $y=x+(h-k)$ for some $h-k \in L$. Ther $(h-k) \bar{\eta}=h \eta-k \eta=0$, and $y \eta+k \eta=(y+k) \eta=(x+h) \eta=$ $x \eta+h \eta=x \eta+k \eta$. Cancelling $k \eta$ gives $x \eta=y \eta$, so that $\phi$ is single-valued. Clearly $\phi$ carries $H \lambda$ onto $H \eta$. Further

$$
(x \lambda+y \lambda) \phi=(x+y) \lambda \phi=(x+y) \eta=x \eta+y \eta=(x \lambda) \phi+(y \lambda) \phi
$$

and

$$
[(x \lambda)(y \lambda)] \phi=(x y) \lambda \phi=(x y) \eta=x \eta y \eta=(x \lambda) \phi(y \lambda) \phi,
$$

so that $\phi$ is a homomorphism. Finally, if $(x \lambda) \phi=(y \lambda) \phi$, then $x \eta=y \eta$ and $x \eta-y \eta=(x-y) \bar{\eta}=0$, so that $x-y \in L$ and $x \lambda=y \lambda$. Hence $\phi$ is indeed an isomorphism. Q.E.D.

COROLlARY A. If $K$ is a subtractive ideal of a halfring $H$ and $v: H \rightarrow$ $H \mid K$ is the natural homomorphism, then $\operatorname{Ker} \bar{v}=R, \bar{H} \mid R$ is isomorphic to $(H \mid K)^{-}$, and $H \bar{v}$ is isomorphic to $H v=H / K$.

PRoof. If $a-b \in \operatorname{Ker} \bar{v}$, then $a v=b v$, and there are $h, k \in K$ such that $a+h=b+k$. Then $a-b=k-h \in \bar{R}$, so that Ker $\bar{\nu}=\bar{R}$. Q.E.D. 
It is now clear that the halfrings $H \lambda$ of Theorem 4 comprise the semiisomorphic images of $H \mid K$. This puts us in a position to determine when all of these semi-isomorphic images are in fact isomorphic images.

THEOREM 5. Let $H$ be a halfring, $K$ a subtractive ideal of $H$. All of the halfring images of $H$ under homomorphisms with kernel $K$ are isomorphic if and only if $K$ is monotypic in $H$.

Proof. Let $K$ be monotypic in $H$ and let $\eta$ be a halfring homomorphism on $H$ with kernel $K$. By Theorem $4, M / K$ is isomorphic to $M / R$ and $M \eta$ is isomorphic to $M / \operatorname{Ker} \bar{\eta}$. But $\operatorname{Ker} \bar{\eta} \in \tau(K)=\{R\}$, so that $M / R$ and $M / \operatorname{Ker} \bar{\eta}$ are identical; thus $M \eta$ is isomorphic to $M / K$.

Conversely, suppose $K$ is not monotypic in $H$. Then there is an $L \in \tau(K)$ such that $L \supset R$. Then $L \mid R$ is a subtractive ideal of $\bar{H} \mid R$ such that $L / R \cap$ $H \mid R=\{R\}$, and the zero ideal of $H \mid R$ is not monotypic. But by Theorem $3 \mathrm{~A}$ and Zorn's Lemma, $\tau(K)$ contains a maximal element; without loss of generality, let this be $L$. Then the zero ideal of $H / L$ is monotypic; otherwise there is a subtractive ideal $I \supset L$ in $\bar{H}$ such that $I / L \cap H / L=\{L\}$, contradicting the maximality of $L$ in $\tau(K)$. Hence $H / R$ and $H / L$ cannot be isomorphic. Q.E.D.

Since the importance of monotypic ideals is now clear, we will introduce here a class of halfrings very well behaved in this respect. A halfring $H$ is called semisubtractive if for every $a, b \in H$ at least one of the equations $a+x=b$ or $a=b+x$ has a solution $x \in H$. Clearly this is equivalent to the condition that $H$ is linearly preordered in the natural preorder of the additive semigroup.

THEOREM 6. In a semisubtractive halfring, every subtractive ideal is monotypic.

Proof. Let $K$ be a subtractive ideal of a semisubtractive halfring $H$, and let $I \in \tau(K)$. Then $R \subseteq I$. Let $i-j \in I$ with $i, j \in H$. If $i+x=j$, then $x=$ $j-i \in I$; if $i=j+x$, then $x=i-j \in I$. In either case $x \in I \cap H=K$. Then $i-j=$ $\pm x \in \bar{R}$, and $I \subseteq \bar{K}$. Hence $\tau(K)=\{R\}$, and $K$ is monotypic. Q.E.D.

4. Further results on types. Since the type of an ideal is a collection of ring ideals, it generates a sublattice of the lattice of all ring ideals in the given difference ring. If $A$ and $B$ are two subtractive ideals, not necessarily in the same halfring, we will say that $\tau(A)$ and $\tau(B)$ are isomorphic if there is a map of $\tau(A)$ onto $\tau(B)$ which extends to a lattice isomorphism of the lattices generated by $\tau(A)$ and $\tau(B)$ respectiveiy. The following mapping theorem is suggested by the proof of Theorem 5 . 
THEOREM 7. Let $\eta$ be a homomorphism of halfring $H$ onto halfring $H^{\prime}$, let $K^{\prime}$ be a subtractive ideal of $H^{\prime}$, and let $K=\eta^{-1}\left(K^{\prime}\right)$. Then $\eta$ induces a lattice isomorphism of $\tau\left(K^{\prime}\right)$ onto the subset of $\tau(K)$ coniaining Ker $\bar{\eta}=I$.

Proof. The map $\bar{\gamma}_{i}: \bar{H} \rightarrow H^{\prime}$ induces a lattice isomorphism $\lambda$ of the lattice of ideals of $\bar{H}$ containing $I$ onto the lattice of all ideals of $\mathrm{H}^{\prime-}$. Let $T \in \tau(K)$, so that $T \cap H=K$, and suppose that $T \supseteq I$. Then $T \lambda=T \bar{\eta}$ is an ideal of $H^{\prime-}$. If $x \in T \bar{\eta} \cap H^{\prime}$, there is an $h \in H$ with $x=h \eta=h \bar{\eta}$, whence $h \in T$ also. Therefore $h \in H \cap T=K$, and $x=h \eta \in K \eta=K^{\prime \prime}$, and $T \lambda \cap H^{\prime} \subseteq K^{\prime \prime}$. Since $K \subseteq T, K^{\prime}=K \eta=K \bar{\eta} \subseteq T \bar{\eta}$, so that $K^{\prime} \subseteq T \lambda \cap H^{\prime}$. Hence $T \hat{\lambda} \in \tau\left(K^{\prime}\right)$.

Conversely, let $T \in \tau\left(K^{\prime}\right)$, so that $T^{\prime} \cap H^{\prime}=K^{\prime \prime}$, and let $T=\bar{\eta}^{-1}\left(T^{\prime}\right)$. Then $I \subseteq T$, If $h \in T \cap H$, then $h \overline{\eta_{i}}=T \bar{\eta}=T^{\prime}$, whence $h \gamma_{j}=h \bar{\eta} \in T^{\prime} \cap H^{\prime}=K^{\prime}$ and $h \in K$. Therefore $T \cap H \subseteq K$. Since $K \subseteq T, T \in \tau(K)$. It follows that the map $\lambda^{-1}$ is the required lattice isomorphisin. QE.D.

COROllary A. If $K$ is monotypic in $H$, then $K^{\prime}$ is monotypic in $H^{\prime}$.

Corollary B. Let $K$ and $T$ be subtractive ideals of a halfring $H$, and let $K \subseteq T$. Then $\tau(T / K)$ in $H / K$ is lattice isomorphic to $\tau(T)$ in $H$. In particular, $T$ is morotypic in $H$ if and only if $T / K$ is monotypic in $H / K$.

Proof. Let $v: H \rightarrow H / K$ be the natural homomorphism. Then Ker $\bar{\nu}=\bar{K}$, and the subset of $\tau(T)$ containing $R$ is all of $\tau(T)$, since $R \subseteq \bar{T}$. Q.E.D.

COROllaRY C. If $K$ is a subtractive ideal of a halfring $H$ such that $H ! K$ is a ring, then $K$ is monotypic in $H$.

Proof. If $H / K$ is a ring, it is semisubtractive, and by Theorem 6 the zero ideal is monotypic. Then $K$ is monotypic in $H$ by Theorem $7 \mathrm{~B}$.

Q.E.D.

We conclude by exploring a relationship between the type of an ideal and the type of a related ideal in the same halfring. For an ideal $K$ of a halfring $H$, let $K^{*}=\{x \in H:(x+H) \cap K \neq \varnothing\}$. Then $K^{*}$ is a subtractive ideal of $H$, called the star of $K$ in $H$. If $K$ is subtractive, $K^{*}$ can be characterized as the largest subsemiring of $H$ such that $K^{*} / K$ is a ring.

THEOREM 8. Let $K$ be a subtractive ideal of a halfring $H$. If $T \in \tau(K)$, then $T+K^{*-} \in \tau\left(K^{*}\right)$.

Proof. First, if $T \in \tau(0)$, then $T+0^{*} E \tau\left(0^{*}\right)$. For $0^{*}$ is a subring of $\bar{H}$, so that clearly $T+0^{*}$ is a subtractive ideal of $\bar{H}$. If $h \in\left(T+0^{*}\right) \cap H$, then $h=t+r$ with $t \in T$ and $r \in 0^{*}$. But then $-r \in 0^{*} \subseteq H$, so that $h+(-r)=t \in H$. Hence $t \in T \cap H=\{0\}$, so that $h=r \in 0^{*}$. Thus $\left(T+0^{*}\right) \cap H \subseteq 0^{*}$. Since clearly $0^{*} \subseteq\left(T+0^{*}\right) \cap H, T+0^{*} \in \tau\left(0^{*}\right)$. 
Now the theorem follows by applying this result to $H / K$ and using Theorem 7B. Q.E.D.

Corollary A. If $K^{*}$ is monotypic, so also is $K$.

Proof. If $T \in \tau(0)$ and $0^{*}$ is monotypic, then $T+0^{*}=0^{*}$, so that $T \subseteq 0^{*} \cap H$ and $T=T \cap H=\{0\}$. Hence 0 is also monotypic. Again the result follows from $H / K$ by applying Theorem 7B. Q.E.D.

\section{REFERENCES}

1. Paul J. Allen, Ideal theory in semirings, Dissertation, Texas Christian University, Fort Worth, Texas, 1967.

2. - A fundamental theorem of homomorphisms for semirings, Proc. Amer. Math. Soc. 21 (1969), 412-416. MR 38 \#5856.

3. S. Bourne, On the homomorphism theorem for semirings, Proc. Nat. Acad. Sci. U.S.A. 38 (1952), 118-119. MR 13, 618.

4. M. Henriksen, Ideals in semirings with commutative addition, Notices Amer. Math. Soc. 5 (1958), 321. Abstract \#542-183.

Department of Mathematics, University of Pittsburgh, Pittsburgh, PennSYLVANLA 15213 